\title{
Mapas de Crisis, como herramienta para mejorar la respuesta en caso de la ocurrencia de un evento de carácter desastroso.
}

\author{
Crisis maps as a tool to improve the response in case of occurrence of an event \\ of disastrous nature. \\ Stephania Suarez Grajales, Juan Mauricio Castaño Rojas \\ Facultad de Ciencias Ambientales, Universidad tecnológica de Pereira, Colombia \\ Correo-e: stsuarez@utp.edu.co
}

\begin{abstract}
Resumen-La gestión del riesgo ha cambiado en las últimas décadas, anteriormente su desarrollo era más una cuestión de expertos, hoy en día incluye la participación pública permanente. Los avances en las tecnológicas de comunicación e información, pueden desempeñar un papel importante en la participación pública e institucional de la gestión del riesgo, especialmente en la respuesta un evento desastroso.

Con el objetivo de mejorar la participación pública en la respuesta a un evento desastroso, se validó una aplicación para celulares Android, en la que se crearon mapas de crisis, con la participación de 50 voluntarios. Los resultados mostraron que los mapas de crisis se pueden crear fácil y rápidamente, como insumo para las organizaciones encargadas de tomar las decisiones durante la ocurrencia del evento desastroso. A partir de esta experiencia se generaron directrices que pueden ser incluidas en los protocolos de respuesta al momento de dar manejo a un evento.
\end{abstract}

Palabras clave-Mapas de crisis, evento desastroso, Gestión del riesgo, participación ciudadana.

Abstract - Risk management has changed in the last decades, previously its development was more a question of experts, nowadays it includes the permanent public participation.

Advances in information and communication technologies can play an important role in public and institutional participation in risk management, especially in response to a disastrous event.

In order to live the public participation in the response to a disastrous event, an application for Android phones was used, in which crisis maps were created, with the participation of 50 volunteers. The results showed that crisis maps can be created easily and quickly, as input for decision-making organizations during the disastrous event. From this experience were generated guidelines that can be included in response protocols at the time of handling an event.
Key Word - Crisis maps, disastrous event, Risk management, citizen participation.

\section{INTRODUCCIÓN}

En América Latina se presenta grandes eventos desastrosos, que afecta especialmente a poblaciones vulnerables, ubicadas en zonas de alto riesgo; en Colombia se estima que desde el 2006 han ocurrido 23.698 desastres de diferentes características [1], entre los cuales se pueden destacar los siguientes: La erupción del volcán Nevado del Ruiz en 1985, cuyos flujos de lodo causaron la muerte de 25 mil personas [2], el terremoto de Armenia en 1999, causo el colapso de miles de edificaciones, con un saldo de 1.185 muertos [3]; y el terremoto de Popayán en 1983, que dejo 1500 víctimas fatales[4]. Todo lo anterior ha dado lugar a que en Colombia se realicen grandes esfuerzos, para la creación y consolidación del sistema nacional de gestión del riesgo, el cual incorpora estrategias de inclusión a las comunidades, reconociéndolas como actores sustantivos de la gestión del riesgo [5].

En el ámbito regional y local, las instituciones que hacen parte del sistema nacional de gestión del riesgo (Gobernaciones, Alcaldías, Cruz roja, Bomberos, Defensa Civil, etc.) parecieran no saber cómo incluir a la sociedad al momento de prevenir, mitigar o manejar un evento desastroso. Esta situación es particularmente evidente al momento de dar un manejo adecuado a la ocurrencia de un evento. Sin embargo, existen casos que muestran que si es posible la participación activa de las personas en el manejo de los eventos desastrosos. Este es el caso del terremoto que afecto puerto príncipe Haití en el año 2010, en este evento un grupo de personas, sin vínculo con institución alguna, uso la georreferenciación de los teléfonos celulares para crear en 48 horas posteriores al evento, mapas de crisis que apoyaron a los organismos de 
respuesta a coordinar la atención inmediata; dirigiendo ayudas a los lugares que priorizaban los mapas de crisis [6].

Los mapas de crisis permiten ver en tiempo real, la evolución del evento en términos de localización de personas afectadas, focalización de las zonas con mayor daño en edificaciones, o cualquier información georreferenciada que se quiera conocer del evento. Este tipo de mapas, creados para diversos temas ameritan el entendimiento espacial de una situación, pudiéndose desarrollar atreves de estrategia que fomenten las formas participativas y descentralizadas de la sociedad civil en la gestión de cualquier crisis.

La primera utilización más común de los mapas de crisis se dio en Kenia en el año 2008, desde las elecciones en ese país se vivió una ola de violencia post electoral, dejando más de mil muertes y un millón de personas desplazadas. En esa crisis un grupo de personas, crearon un sitio web por medio de google, para que cualquier persona con un teléfono móvil o acceso a internet, registrara cualquier tipo de abuso contra los derechos humanos; los mapas de crisis generados en ese momento, lograron concientizar a las personas del resto del mundo sobre lo vivido en ese lugar, además se generaron ayudas por medio de organización sin ánimo de lucro [7].

Por otra parte en el año 2011, en la Guerra de Libia; se creó la página de google "Libya Crisis Maps" auspiciada por la oficina de las naciones unidas para la coordinación de asuntos humanitarios (UNAOCHA), esta página mostraba mapas de crisis con distintos tipos de emergencias y áreas mas afectadas por la violencia [8].

Pero en definitiva el caso más relevante fue la "misión 4636", en el terremoto de puerto principal Haití, dicha misión consistió en generar mapas de crisis por medio de la misma plataforma web en la que se desarrollaron los mapas en Kenia llamada Ushahidi, los mapas fueron impulsados por Patrick Meier, quien desde su domicilio, empezó a generar los mapas de crisis por medio de una red de voluntarios que traducían al inglés los reportes generados desde Haití en creole, posteriormente Usahidi se vinculó a UNDAC (Coordinación y evaluación de desastre), esta organización era la encarga de enviar los equipos de búsqueda y recate con mayores necesidad, en ese sentido la cooperación con Usahidi sirvió para guiar a los socorristas de las naciones unidad, el comando sur de marines de los Estados Unidos a facilitar la llegada a las victimas los alimentos, agua y los servicios de salud [6].

Por último, la sociedad geológica de los estados unidos está creando una herramienta llamada "Twitter Earthquake Detector TED" la cual busca trinos relacionados con la ubicación y la gravedad de los daños al momento de un terremoto, y a partir de esa información elabora un mapa de tendencias y patrones, con los cuales se podría dar una atención más dirigida y rápida al desastre [6].
En ese sentido, el propósito del estudio fue validar la utilización de una aplicación para celulares Android que permitiera generar mapas de crisis al momento de un evento de carácter desastroso en la ciudad de Pereira, Colombia.

Logrando una participación ciudadana por medio de aportes georreferenciados de localización de personas afectadas y daño en edificaciones, que contribuya al manejo y la recuperación de un evento desastroso.

De acuerdo a lo anterior presento las tecnologías de información y comunicación, como una herramienta de gestión, la cual permite la participación ciudadana en el manejo de eventos desastrosos; aportando a sistemas de información georreferenciados, que contribuyan al manejo y la recuperación del evento por medio de la creación colectiva de mapas de crisis.

\section{METODOLOGIA}

La investigación se desarrolló en tres etapas, siguiendo la metodología de investigación holística [9], en la cual; la etapa descriptiva se define como la descripción del contexto del tema a estudiar, en este caso la descripción de las herramientas disponibles en la ciudad de Pereira para dar respuesta oportuna ante la ocurrencia de un evento desastroso.

Para eso se solicitó información a las entidades más representativas que conforman el comité municipal para el manejo de desastres y calamidad en la ciudad de Pereira, enmarcadas en el decreto $\mathrm{N}^{\circ} 605$ del 17 de julio del 2012, las cuales son:

1. Dirección operativa para la prevención y atención de desastres.

2. Secretaria de planeación.

3. Secretaria de salud y seguridad social.

4. Instituto Colombiano de bienestar familiar.

5. Batallón San Mateo

6. Policía metropolitana de Pereira.

7. Defensa civil.

8. Cruz roja

9. Cuerpo de bomberos

La información suministrada por las entidades correspondió a describir el deber ser, el accionar de cada una de ellas frente a la ocurrencia de cualquier evento de carácter desastroso y la fuente de financiación de sus actividades misionales.

Por otra parte, se realizó una revisión histórica de la normatividad y los esfuerzos a nivel gubernamental e institucional para generar una política nacional de gestión del riesgo de desastres.

La Segunda etapa de la investigación es la etapa interactiva; esta consiste en el diseño de la investigación y la recolección de datos, en esta etapa se pone en práctica la elaboración de diferentes mapas de crisis por medio de dos simulacros de 
sismos, cuyo efecto se focalizo en los predios de la Universidad Tecnológica de Pereira. A continuación, se describen las actividades realizadas para la realización de cada simulacro.

Como primera medida se realizó la selección de la aplicación App: por medio de la metodología AHP (análisis multicriterio), entre 4 aplicaciones para sistema operativo Android (Micromappers, Crowmap, Yo reporto, Memento Database) las características evaluadas para la selección fueron: la facilidad de uso, accesibilidad, su capacidad de capturar coordenadas geográficas y su gratuidad, en este análisis la aplicación Memento database fue la seleccionada para el desarrollo de la investigación.

Para el registro de la información en los simulacros; se crearon formularios que contenían preguntas como: el nombre de cada persona, la selección de una clasificación de daño a una edificación desde leve, moderado, fuerte, hasta colapso total [10], la selección de una afectación humana como prioridad 1, 2,3, o 4 [11], además se debía tomar una fotografía al sitio y por ultimo georreferenciar el lugar en donde se encontraba al momento de registrar el formulario, la hora y fecha; y opcionalmente la persona podía enviar una nota de voz.

Posteriormente se realizó el primer simulacro con el propósito de validar la capacidad de captura de información de la aplicación seleccionada para la investigación. En él participaron diez voluntarios, los cuales registraron en la aplicación Memento Database, la información visualizada en una fotografía qué contenía efectos potenciales de un evento de carácter desastroso.

Como segunda medida se preparó el simulacro final, con el diseñó de un mapa hipotético de daños en edificaciones y afectaciones humanas después de un evento desastroso asociado a un movimiento telúrico de gran magnitud.

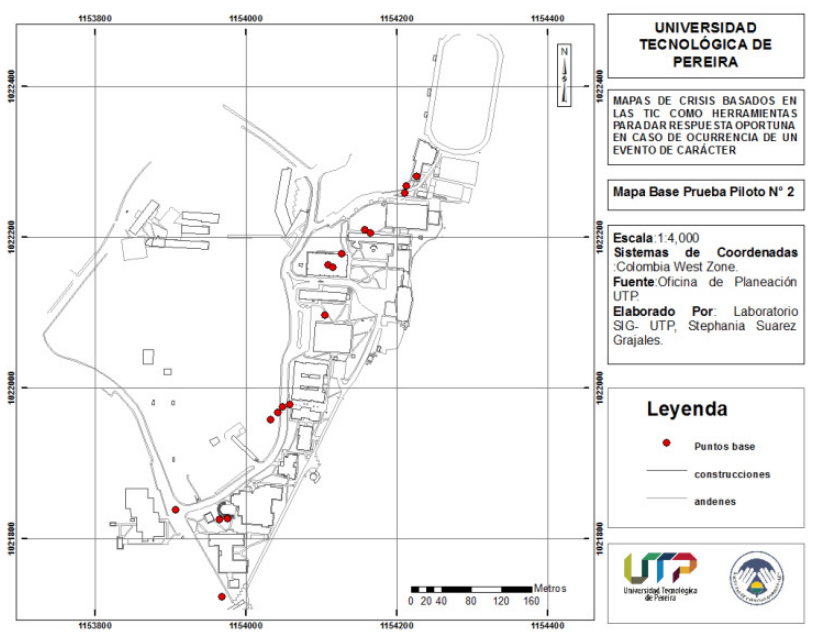

Figura 1. Mapa base, creado para el registro información del simulacro final.
Además de lo anterior, para la realización del simulacro final, se generó un código de colores que representaba daños en edificaciones o afectaciones en vida humana; este código de colores se materializó en el simulacro mediante la ubicación de cintas de colores en diferentes lugares del campus Universitario de la Universidad Tecnológica de Pereira.

Es decir, en el campus universitario se ubicaron cintas de colores que representaban los daños en los lugares previamente escogidos con el mapa hipotético de daños en edificaciones y vida humana.

De otra parte, se realizó la capacitación de 50 personas en la utilización de la aplicación Memento Database, en la importancia de la participación en el manejo de desastres en la gestión del riesgo, y en la participación de la construcción colectiva de mapas de crisis.

Finalmente, la tercera etapa de la investigación es una etapa propositiva, en esta se interpreta la información recogida por las instituciones que conforman el comité municipal para el manejo de desastres y calamidad en Pereira, y se analiza la experiencia con los simulacros realizados para la realización de los mapas de crisis. Con el fin de proponer lineamientos normativos y operativos contribuyan a la inclusión de los mapas de crisis como herramienta en el manejo de un evento de carácter desastroso en la ciudad de Pereira.

\section{RESULTADOS}

\section{Fase descriptiva:}

En la descripción de las herramientas disponibles en la ciudad de Pereira para dar respuesta oportuna a la ocurrencia de un evento desastroso; se encontró que en Colombia se ha hecho un gran esfuerzo para la creación de la política nacional de gestión del riesgo mediante la ley 1523 del 2012 y a nivel municipal se acoge la política mediante el decreto 605 del 17 de julio del 2012; por el cual se conforma y organiza el consejo municipal de gestión del riesgo del municipio de Pereira, los comités municipales y se dictan otra disposiciones.

Sin embargo de acuerdo a la búsqueda de información y algunas charlas realizadas con funcionarios de las entidades municipales; se halló que el decreto 605 del 2012 no se aplica de manera correcta, ya que los funcionarios no están enterados de que se encuentran en el comité municipal de gestión del riesgo de desastres, ni cuál es su proceder en el comité municipal para el manejo de desastres y calamidades, además se evidencio el desconocimiento de los protocolos y su deber misional ante la ocurrencia de un evento de carácter desastroso.

Por otra parte en los protocolos de respuesta presentados por cada entidad están desarticulados con el protocolo general 
designado por la dirección operativa para el manejo de desastres y calamidades DOPAD, para la ciudad de Pereira y por supuesto que en ningún protocolo reconoce el uso de herramientas participativas y menos de la creación de mapas de crisis como una herramienta al momento de dar respuesta a un evento de carácter desastroso.

\section{Fase Interactiva:}

De acuerdo al diseño de la investigación y la recolección de datos, en esta etapa se pone en práctica la elaboración de diferentes mapas de crisis por medio de dos simulacros de sismos cuyo efecto se focalizo en los predios de la Universidad Tecnológica de Pereira.

Para empezar se pudo evidenciar gran diversidad de aplicaciones existentes para aportar al manejo del desastre mediante la ocurrencia de evento de carácter desastroso, como lo son: Crowmap, Crisismap, Yo Reporto, Memento Database, entre otros; dichas aplicaciones se han utilizado en desastres ocurridos en todo el mundo debido a la necesidad de generar información geográfica rápidamente para dar una mejor respuesta en la ocurrencia de evento de carácter desastroso.

Finalmente se escogió la aplicación Memento Database por tener mayor acogida en los criterios de accesibilidad, impacto, visualización de mapas, y administración del software.

En consecuencia, se procedió a realizar el simulacro $\mathrm{N}^{\circ} 1$, para la verificación de la aplicación Memento Database en el registro de datos; como resultado de la prueba fue el registro de 7 formularios, principalmente desde la Universidad Tecnológica de Pereira.

El simulacro $\mathrm{N}^{\circ} 1$, comprobó la viabilidad de la aplicación seleccionada para la recolección de datos y la creación colectiva de los mapas de crisis, además la prueba sirvió para mejorar la capacitación en la importancia de este tipo de procesos para el manejo de desastres en la gestión del riesgo y se sugirió mejorar el formulario propuesto para la creación de mapas de crisis con el fin de que los voluntarios registraran más rápidamente la información. A continuación, presento la imagen de los puntos registrados por los voluntarios de la prueba $\mathrm{N}^{\circ}$ 1:

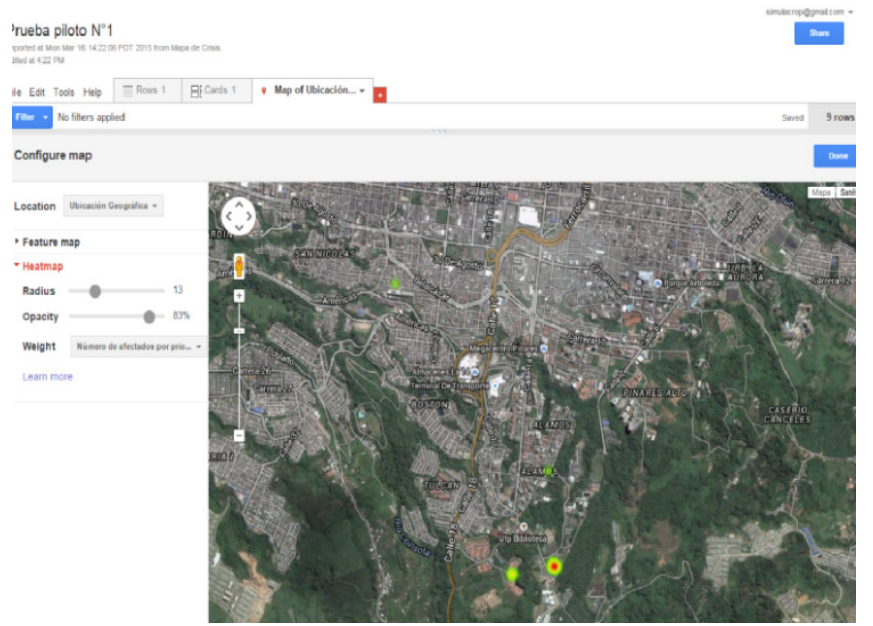

Figura 2. Imagen de calor, resultado de la prueba piloto $\mathrm{N}^{\circ} 1$

Finalmente, en la fase interactiva se realizó el simulacro $\mathrm{N}^{\circ} 2$, en el cual generaron 7 mapas de crisis, con un total de 69 registros realizados por los voluntarios; en los mapas se evidencia; que a medida que pasa en tiempo del simulacro (24 horas) se alimenta la base de datos de acuerdo al mapa base propuesto inicialmente. A continuación, presento 4 mapas en los cuales se evidencia un cambio significativo en el tiempo:

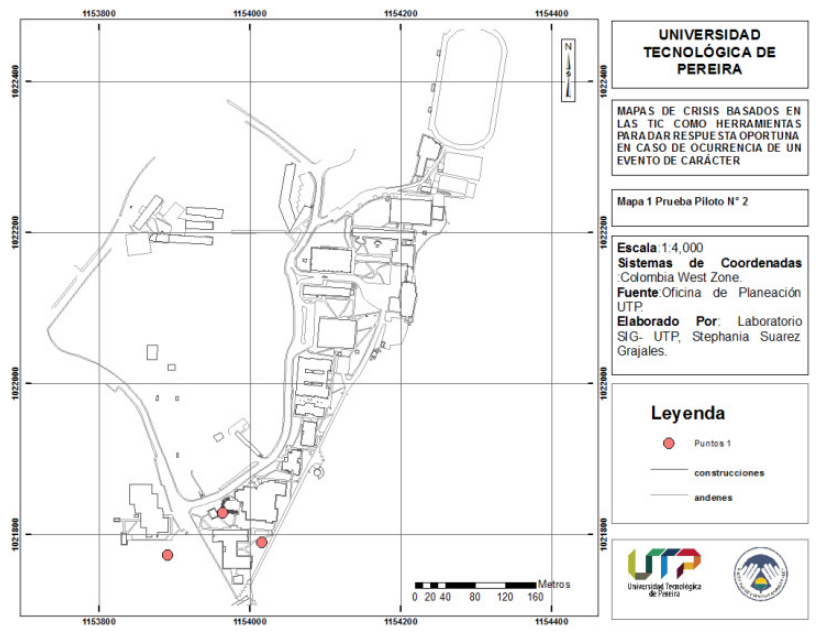

Figura 3. Mapa 1, resultado de la prueba $\mathrm{N}^{\circ} 2$ 


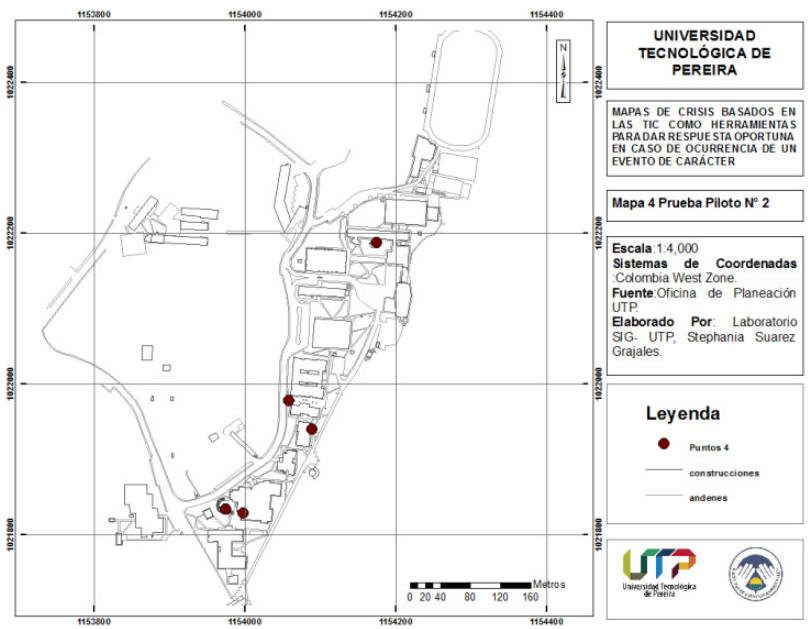

Figura 4. Mapa 4, resultado de la prueba $\mathrm{N}^{\circ} 2$

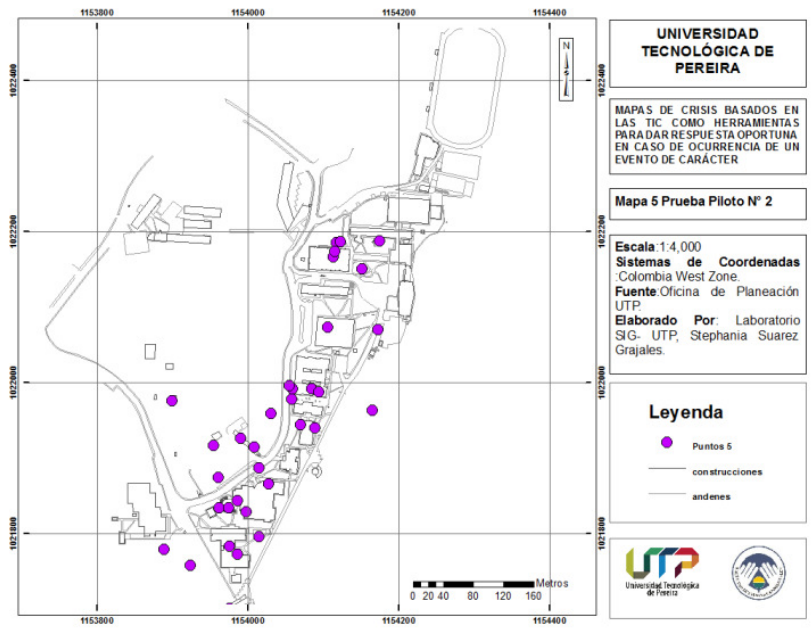

Figura 5. Mapa 5, resultado de la prueba $\mathrm{N}^{\circ} 2$

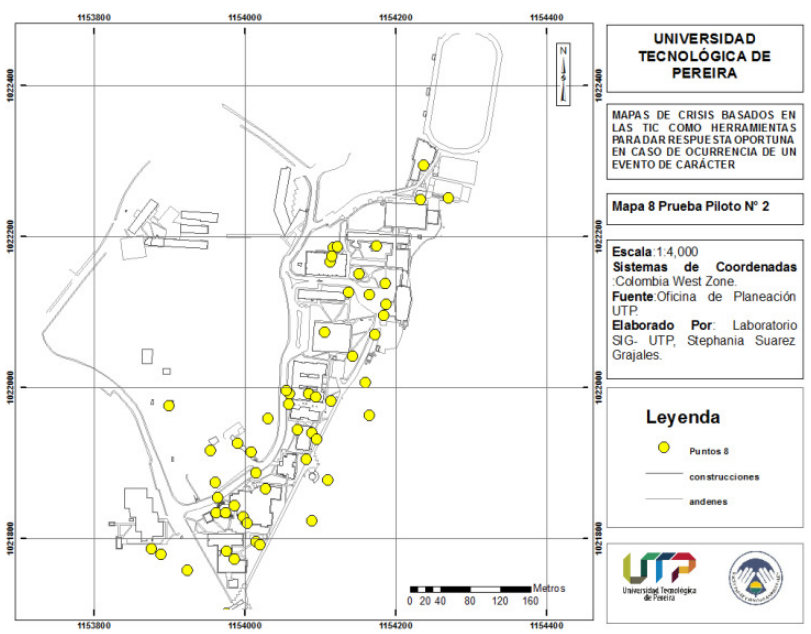

Figura 6. Mapa 8, resultado de la prueba $\mathrm{N}^{\circ} 2$.

Por último, se generó un mapa contraste, en el cual se realizó una comparación del mapa base, y el mapa final realizado por los voluntarios; con el fin de verificar si se registraron los puntos propuestos en la prueba $\mathrm{N}^{\circ} 2$.

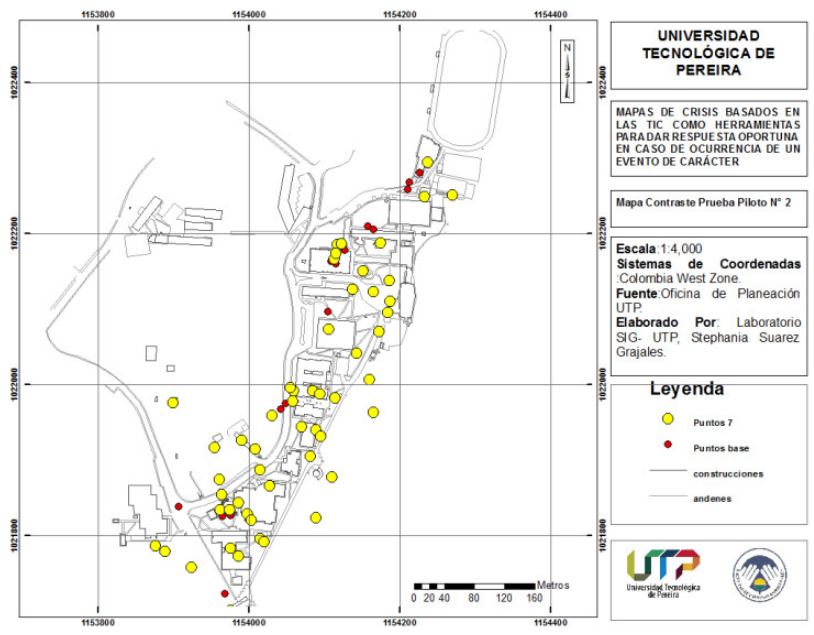

Figura 7. Mapa contraste, resultado de la prueba $\mathrm{N}^{\circ} 2$ Fuente:

Los mapas de crisis arrojaron unos puntos prioritarios de atención a la emergencia, como se planteó en el mapa base, los puntos representados en imágenes de calor son los siguientes:

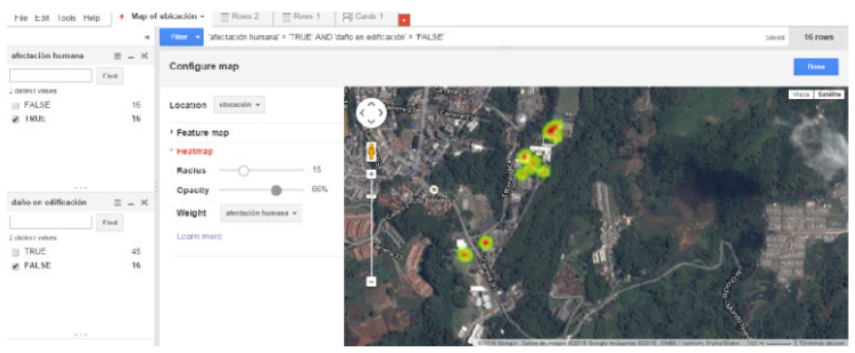

Figura 8. Imagen de calor, resultado de la prueba $\mathrm{N}^{\circ} 2$ en afectación a vida humana

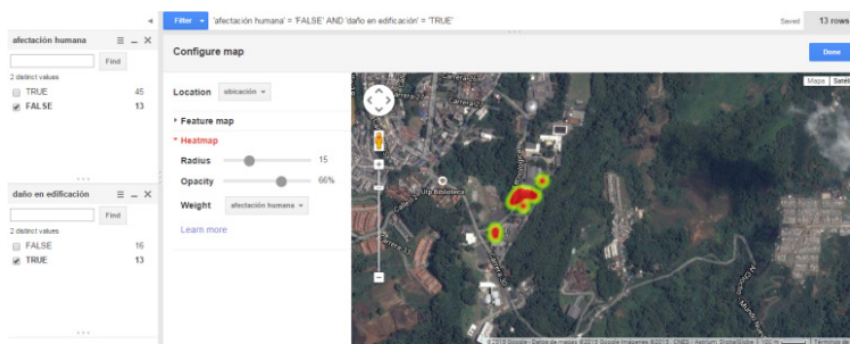

Figura 9. Imagen de calor, resultado de la prueba $\mathrm{N}^{\circ} 2$ en daño en edificaciones.

\section{Fase Propositiva:}

\section{Lineamientos Normativos:}

Se formularon lineamientos normativos para la creación colectiva de mapas de crisis, los lineamientos son enfocados a la articulación de los mapas de crisis en el comité municipal 
para el manejo de desastres y calamidad, y en la creación de un comité delegado para la articulación de los mapas de crisis en los protocolos de respuesta de cada entidad para la toma de decisiones.

Los lineamientos normativos permiten incluir a la comunidad de forma participativa en la toma de decisiones frente al territorio ante la ocurrencia de un evento desastroso, no obstante todos los procesos que se desarrollen con el objetivo de mejorar la gestión del riesgo deben ser procesos constante de capacitación y de mejoras en la aplicación que conlleve a la creación colectiva de los mapas de crisis.

1. Monitoreo continuo de los mapas de crisis, por parte del comité municipal para el manejo de desastres y calamidades.

El presente lineamento se basa en crear una nueva función al comité, en la cual se monitoreen continuamente los mapas de crisis generados por la comunidad en caso de cualquier evento.

Los mapas de crisis se podrían convertir en una guía para la toma de decisiones en las acciones reactivas y correctivas, ante la ocurrencia de cualquier evento de carácter desastroso.

El monitoreo constante podrá generar una base datos en los que se localicen las zonas más afectadas en la ciudad de Pereira para la toma de decisiones con el fin de evitar futuros desastres.

2. Crear un protocolo actualizado para el manejo de un desastre en la ciudad de Pereira, incluyendo los mapas de crisis en la atención por parte del comité municipal para el manejo de desastres y calamidades.

El lineamiento está enfocado a la realización de un protocolo especial para el manejo de un evento de carácter desastroso, de acuerdo a la creación colectiva de mapas de crisis. Para el cumplimiento del lineamiento se propone la conformación de un equipo conformado por un delegado de la cruz roja (especialista en triage), un bombero, un rescatista de la defensa civil, y un administrador ambiental especialista en gestión del riesgo, dicho lineamiento se solicita debido a la necesidad del comité municipal en manejar los mapas de crisis para orientar la respuesta en caso de ocurrencia de un evento de carácter desastroso, generando lineamientos de utilización de los mapas al momento del evento.

Estos lineamientos estarán enfocados a incluir los mapas de crisis para informar la situación por la cual este pasando el municipio de Pereira, teniendo en cuenta que comunidad está alimentando constantemente los mapas de crisis al momento de un evento desastroso.

\section{Establecer mayor operatividad en el consejo municipal de gestión del riesgo.}

El lineamiento está enfocado a fomentar la participación y apropiación de las instituciones pertenecientes al consejo municipal de gestión del riesgo, a partir de metas por implementar en la ciudad de Pereira en cuanto a la gestión del riesgo, incluyendo actividades como:

- Criterios y estándares de calidad en la gestión del riesgo.

- Estudios técnicos.

- Control para proyectos de ocupación, intervención y adecuación de terrenos.

- $\quad$ Escenarios de recuperación y reconstrucción antes de los desastres.

- Participación ciudadana.

\section{Lineamientos Operativos:}

Se crean lineamientos de carácter operativo para fomentar el uso y la creación de los mapas de crisis en la ciudad de Pereira, mediante la creación de una aplicación exclusiva para la realización de los mapas de crisis al momento de un evento de carácter desastroso.

Es necesario la inclusión de los mapas en los protocolos de respuesta destinados por la dirección operativa para la prevención y atención de desastre- DOPAD.

Finalmente se crea un lineamiento para dar capacitación continua a personas, especialmente relacionadas con el comité municipal para el manejo de desastres y calamidad en gestión del riesgo.

Se toma como premisa que cualquier estado de la gestión del riesgo es un proceso social y por lo tanto los mapas de crisis como herramienta en la parte reactiva de la gestión del riesgo, son concebidos por la sociedad, para la sociedad, y el éxito de la creación de los mapas de crisis es lograr una capacitación continua y una sensibilización ante este tipo de procesos que ayudan a la mejora en la respuesta de determinado evento.

1. Crear una aplicación gratuita para Android enfocada a la creación colectiva de mapas de crisis al momento de un evento de carácter desastroso en la ciudad de Pereira.

Se propone existencia de una aplicación exclusiva para la elaboración colectiva de mapas de crisis al momento de un evento de carácter desastroso en la ciudad de Pereira, la aplicación puede ser única para el apoyo de organismos de respuesta en el manejo del desastre.

El lineamiento se planteó debido a que Memento Database fue la herramienta seleccionada para probar la utilidad de los mapas de crisis al momento de un evento de carácter desastroso, sin embargo, esta tiene unos límites de sincronización de librerías gratuitas, por eso una aplicación exclusiva para el tema podría ser un gran aporte a la 
ciudadanía; con el fin de estar mejor preparados para aportar a la respuesta ante cualquier evento.

La aplicación deberá ser creada para celulares Android con la finalidad exclusiva de registrar los impactos ocurridos en la ciudad, después de un evento de carácter desastroso.

Como la aplicación requiere un monitoreo constante debe ser administrada desde un servidor base en el que se elaboren los mapas de crisis, y se corrobore continuamente la información reportada en la herramienta exclusiva para la creación de mapas.

En la ciudad de Pereira se propone que la administración de la aplicación para el monitoreo de los mapas de crisis, sea realizado por medio de la red hidroclimatológica del departamento de Risaralda; ya que cuenta con los equipos necesarios y la operatividad para realizar un monitoreo las veinticuatro horas al día.

Además, se podrían generar la articulación de los monitoreos de las variables hidroclimatológicas con los mapas de crisis en la ocurrencia de cualquier tipo de evento ya sea de carácter desastroso o no. Por ejemplo, un deslizamiento o una avenida torrencial.

Por otra parte el administrador deberá encargarse del fomento y la educación continua a los ciudadanos de Pereira en la aplicación creada para la realización de los mapas de crisis, y en la gestión del riesgo en general.

2. Incluir los mapas en la respuesta por parte de la Dirección Operativa para la Prevención y Atención de Desastres (DOPAD) en la ocurrencia de un evento desastroso.

El lineamiento se basa en la inclusión de los mapas de crisis al momento de dirigir el escuadrón de socorro en el momento de un evento de carácter desastroso.

De esta manera se estaría incluyendo a la comunidad en la respuesta, teniendo en cuenta que en cada centro de evaluación zonal (CEZ) Exista un escuadrón de socorro "Comité para la respuesta oportuna" enfocado especialmente para dar respuesta oportuna mediante la utilización de mapas de crisis.

\section{Capacitación a la comunidad en general para la elaboración colectiva de mapas de crisis.}

Las entidades que hacen parte del comité municipal para el manejo de desastres y calamidades deberán estar informadas y listas para participar en el manejo de evento de carácter desastroso, por tal motivo es necesario implementar programas internos de capacitación para sus colaboradores, asegurando que además estén en constante capacitación en gestión del riesgo, primeros auxilios.
Por otra parte, los funcionarios deben estar preparados con sus celulares para la elaboración colectiva de mapas de crisis al momento de un evento de carácter desastroso.

Los funcionarios que hacen parte del consejo municipal de gestión del riesgo pueden servir de gran ayuda en la creación colectiva de los mapas de crisis y deben ser los más capacitados en el tema.

Otra utilidad para este lineamiento es el apoyo en la articulación de los organismos de respuesta y sociedad en general, debido a que cada funcionario o persona capacitada puede estar en la voluntad de capacitar a otra persona para la creación de colectiva de mapas de crisis.

\section{CONCLUSIONES}

* Este tipo de trabajo deben estar en la búsqueda de mejorar los esfuerzos para el bienestar de la sociedad, potenciando la capacidad de resiliencia de la sociedad al momento de un evento de carácter desastroso, por tal motivo se debe recuperar una cultura del riesgo local, que sea participativa e incluyente con los ciudadanos.

* Es necesario que se utilice una tecnología apropiada y exclusiva para la realización de mapas de crisis al momento de un evento desastroso en la ciudad de Pereira potenciando los recursos sociales, educativos, comerciales y tecnológicos para responder y prevenir futuros desastres.

* Los procesos descritos en el presente trabajo deben ser participativos, ya que el objetivo es la formación de una red de voluntarios que trabajando juntos contribuya al conocimiento y la reducción del riesgo en situaciones de emergencia. Promoviendo una innovación de participación social con la articulación entre instituciones y comunidad al momento de una emergencia que abordada problemas complejos que conlleva dicha situación.

* En la presente investigación se puede apreciar que la gestión del riesgo es un proceso continuo, interdisciplinario e interinstitucional. En la ciudad de Pereira que por recorrer un camino largo para lograr dichos enfoques, ya que no solo basta con las normas propuestas, la gestión del riesgo es más un proceso de un intencionalidad y disposición de las instituciones y la ciudadanía.

* El presente trabajo se enfoca a generar un aumento de resiliencia en caso de ocurrencia de un evento desastroso, por tal motivo es necesario crear procesos desde ahora para aumentar dicha resiliencia, teniendo en cuenta que esta además de ser una meta, es un camino constante por construir día a día, mejorando las prácticas y políticas de gestión y basándose en lecciones aprendidas. 
*El riesgo es un elemento inherente a la manera como pensamos y ejecutamos los modelos de desarrollo, por tal motivo se debe establecer una imagen objetivo de la gestión del riesgo y de acuerdo a eso crear los modelos de desarrollo; integrando siempre la gestión del riesgo con la gestión del desarrollo.

* Como se puedo evidenciar, los mapas de crisis son una herramienta útil para que los organismos de respuesta puedan priorizar situaciones al momento de un evento de carácter desastroso, Pero es necesario aclarar que no se deben suspender los protocolos de respuesta de cada uno de los organismos de socorro por lo mapas de crisis, al contrario este tipo de herramientas son útiles para fortalecer y articular el accionar ya previsto, generando más dinamismo al momento de aportar al manejo del desastre.

*Teniendo en cuenta las mejoras y recomendaciones que se enuncian en los lineamientos generales para la creación colectiva de mapas de crisis; la experiencia en general ha sido satisfactoria ya que a mi entender desde la interdisciplinariedad que conllevan las ciencias ambientales se pueden hacer aportes significativos a la sociedad, involucrándola de una manera tan sencilla y provechosa en la parte reactiva de la gestión del riesgo.

*Por último se concluye que la institucionalidad en la gestión del riesgo para la ciudad de Pereira, requiere mayores esfuerzos para la articulación de procesos que conlleven a una mejora en la capacidad de respuesta institucional al momento de un evento de carácter desastroso. Ya que aunque se realicen esfuerzos normativos para la creación de un política enfocada a la gestión del riesgo, dicha política se debe articular con los planes de desarrollo a nivel municipal y por ende con las instituciones que hacen parte del consejo municipal de gestión del riesgo.

\section{RECOMENDACIONES}

Agradecimientos a las entidades que hacen parte del comité municipal para el manejo de desastre y calamidades en la ciudad de Pereira, por el suministro de la información.

Al grupo de investigación en Ecología, ingeniería y Sociedad de la Universidad Tecnológica de Pereira, por su participación y apoyo en la investigación.

A los voluntarios que participaron en los simulacros para la realización de mapas de crisis en la Universidad Tecnológica de Pereira.

\section{REFERENCIAS}

[1] AGUILAR, Ana María; BEDOYA, Geovany; HERMELIN, Michel. INVENTARIO DE LOS DESASTRES DE ORIGEN NATURAL EN COLOMBIA, 1970 2006-
Limitantes, tendencias y necesidades futuras. Gestión y Ambiente, 2008, vol. 11, no 1, p. 109-120.

[2] Escobar, Gonzalo Duque. 2010. bdigital UNAL. Las lecciones del Ruiz a los 25 años. [En línea] 09 de 11 de 2010. [Citado el: 26 de 10 de 2014.]

http://www.bdigital.unal.edu.co/2281/.

[3] LÓPEZ, Carlos Alberto Baena. Retos de Colombia frente a la gestión del riesgo de desastre natural. En Revista Forum. Universidad Nacional de Colombia, sede Medellín. Departamento de Ciencia Política, 2011. p. 91-108.

[4] Gómez, Ney Guzman. 1984. Biblioteca Virtual em saúde . persquisa em base de dador. [En línea] 1984. [Citado el: 26 de 10 de 2014.] http://bases.bireme.br/cgi-

bin/wxislind.exe/iah/online/?IsisScript=iah/iah.xis\&src=googl e \&base $=$ LILACS\&lang $=$ p\&nextAction $=\operatorname{lnk} \&$ exprSearch $=132$ 648\&indexSearch=ID.

[5] Congreso de la republica. (2012, 04 24). Federación internacional de sociedades de la cruz roja y de la media luna roja. Retrieved from ley 1523 del 24 de abril del 2012: http://www.ifrc.org/docs/idrl/1057ES.pdf

[6] ZOLLI, Andrew, Resilience Why Things Bounce Back, Vol 1. Bogota, Buena semilla, 2012.

[7] MEIER, Patrick. Crisis mapping in action: How open source software and global volunteer networks are changing the world, one map at a time. Journal of Map \& Geography Libraries, 2012, vol. 8, no 2, p. 89-100.

[8] BALCELL, Marta Poblet. Móviles, mapas, satélites y redes sociales: gestión de crisis 2.0. Lychnos, 2011, no 7, p. 75-79.

[9] HURTADO DE BARRERA, Jacqueline. Metodología de la investigación holística. Fundacite-SYPAL. Caracas, 1998.

[10] Presidencia de la republica. (2010). Decreto 0926 del 2010. Retrieved from Reglamento Colombiano de Construccion sismoresistente: http://www.minambiente.gov.co/images/normativa/d ecretos/2010/dec_0926_2010_diario_oficial.pdf.

[11] GÓMEZ JIMÉNEZ, J.; RAMÓN-PARDO, P.; RUA MONCADA, C. Manual para la implementación de un sistema de triaje para los cuartos de urgencias. Washington, DC. Organización Panamericana de la Salud. Organización Mundial de la Salud. OPS/OMS. 2011.(Consultado 22 agosto 2011) 